\title{
Dynamic Modeling and Simulation Analysis for Stratospheric Airship
}

\author{
Yi ZHANG ${ }^{1, a}$, Longbin LIU ${ }^{1, b}$ \\ ${ }^{1}$ School of Aeronautic Science and Engineering, Beijing University of Aeronautics and Astronautics, \\ 37 Xueyuan Road, Beijing 100191, China \\ aemail: zhangyi8804@126.com, bemail: longbuaa@163.com
}

Keywords: Dynamic Modeling; Simulation Analysis; Stratospheric Airship

\begin{abstract}
A complete six-degree-of-freedom dynamic model is addressed for a stratospheric airship. Particular characteristics of the stratospheric airship are introduced. Besides, the airship's equations of motion are constructed by including the factors about buoyancy, aerodynamic force, and added mass. Based on the model, Dynamic stability analysis, control and response simulation of airship are accomplished. The results show that stratospheric airship is stable both in longitudinal and lateral direction within specific bounds of attitude angles. With the control surface and thrust, stratospheric airship is local controllable. The presented model is feasible and it call be used in engineering practice.
\end{abstract}

\section{Introduction}

With the accumulation of knowledge and statistics data about stratospheric layer, it is proved that stratosphere is the most peaceful layer in atmosphere with a stead wind and has the protection of mesosphere and ionosphere. Therefore, in recent years, the trend of developing stratospheric platform springs up over a lot of countries [1].

Paiva [2] designed a robust PID controller for airship attitude based on the linear simplified model, the controller combines the robust pole placement techniques to ensure the performance of the closed-loop system response. Acosta [3] designed position PD controller and velocity dynamic inversion controller for Titan airship using feedback linearization method. Hygounenc [4] designed longitudinal motion controller for speed and pitch control, and the result of simulation is presented. Trevino [5] conducted a stability controller for a Tri-Turbofan airship using receding horizon control method.

This paper introduces a modeling method for stratospheric airship. Based on the model, dynamic stability analysis, response of control and the simulation results are presented.

\section{Modeling of Stratospheric Airship}

The dynamic modeling method for stratospheric airship is similar to traditional aircraft dynamic model which is addressed from momentum theorem and angular momentum theorem [6]. The most particular property being different from the traditional aircraft is that airship is a kind of buoyancy vehicle. Therefore buoyancy, additional inertia forces and other particular properties should be considered in modeling. To develop a meaningful mathematical model of the airship, it is first necessary to make some assumptions which constrain the problem to practical bounds and which help to provide dynamic visibility by reducing the equations of motion to a reasonable simple level. The assumptions are:

- The familiar aircraft dynamic modeling methods apply.

- Rigid body motion only is considered, aeroelastic effects are omitted.

- The airship is symmetric about $O x z$ plane, both gravity center and centroid lie in that plane.

- The mass of the airship remains constant.

The layout of the airships is classical. It has four mutually perpendicular rear fin surfaces, each incorporating an aerodynamic flap-type control surface, and it has two independently controlled thrust vectoring propulsion units mounted either side of the aft end of the gondola. The structure 
diagram of the stratospheric airship is shown in Figure 1.

As in typical aircraft practice, it is convenient to define a right-handed orthogonal axis system fixed in the airship and constrained to move with it. Two coordinate systems are defined in this paper. One is the inertial coordinate system $O x_{g} y_{g} z_{g}$ with the origin at arbitrary point on the ground, the $O x_{g}$ axis coincident with north and the $O z_{g}$ axis pointing in the center of the Earth. The other is the body coordinate system $O x y z$ with the origin at the center of volume $(C B)$, the $O x$ axis coincident with the axis of symmetry of the envelop, and the $O x z$ plane coincident with the longitudinal plane of symmetry of the airship.

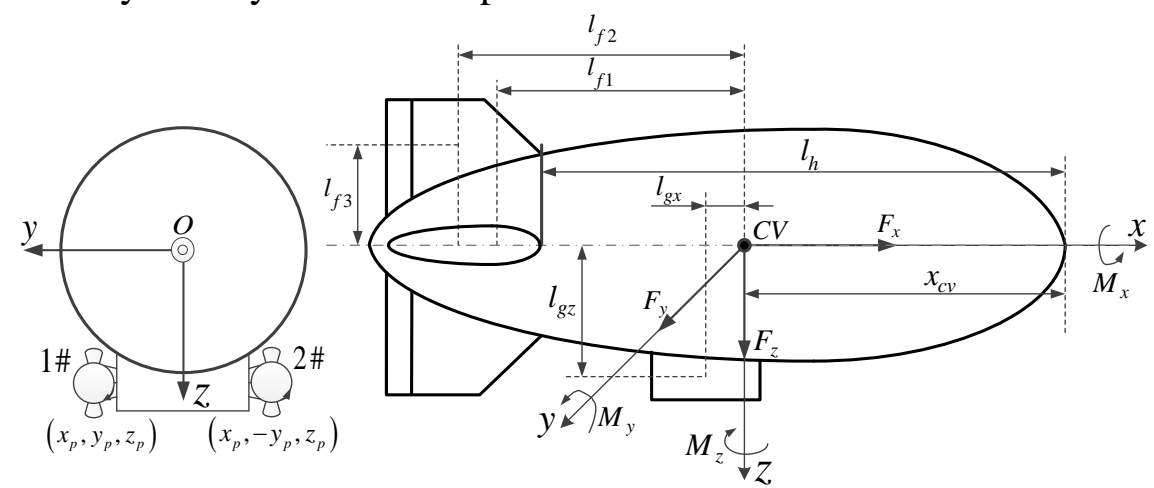

Fig.1. Structure diagram of the stratospheric airship

Due to the fact that airship is a class of Lighter-than-air vehicle, the force situation of airship is more complex. Main forces acting on the airship include gravity, buoyancy, aerodynamic forces, thrust from propeller and additional inertia forces.

Gravity and buoyancy are both exist in $O z_{g}$ axis in inertial coordinate system $O x_{g} y_{g} z_{g}$. And due to the center of buoyance is at the origin of Oxyz, there are no torques from buoyancy, so $\boldsymbol{M}_{B}=[0,0,0]^{\mathrm{T}}$.

$$
\begin{aligned}
& \boldsymbol{F}_{G}=\boldsymbol{R}_{g b}[0,0, G]^{\mathrm{T}}, \quad \boldsymbol{F}_{B}=\boldsymbol{R}_{g b}[0,0,-B]^{\mathrm{T}} \\
& \boldsymbol{M}_{G}=\left[\begin{array}{ccc}
0 & -z_{c} & y_{c} \\
z_{c} & 0 & -x_{c} \\
-y_{c} & x_{c} & 0
\end{array}\right] \boldsymbol{F}_{G}, \quad \boldsymbol{M}_{B}=[0,0,0]^{\mathrm{T}}
\end{aligned}
$$

where $\boldsymbol{F}_{G}$ and $\boldsymbol{F}_{B}$ are gravity and buoyancy in body coordinate system, $\boldsymbol{M}_{G}$ is torques caused by gravity, $\boldsymbol{R}_{g b}$ is transition matrix from $O x_{g} y_{g} z_{g}$ to $O x y z,\left[x_{c}, y_{c}, Z_{c}\right]$ is the coordinate values of gravity in Oxyz. G and B are values of gravity force and buoyancy force, respectively.

The aerodynamic forces acting on the airship can be formulated as principal vector and principal moment in body coordinate system. According to the appearance of airship shown in Figure 1, Mueller put forward a simplified estimation method based on wind tunnel test data.

$$
\begin{aligned}
& X_{a}=-Q_{\infty}\left[C_{X 1} \cos ^{2} \alpha \cos ^{2} \beta+C_{X 2} \sin (2 \alpha) \sin (\alpha / 2)\right] \\
& Y_{a}=-Q_{\infty}\left[C_{Y 1} \cos (\beta / 2) \sin (2 \beta)+C_{Y 2} \sin (2 \beta)+C_{Y 3} \sin \beta \sin (|\beta|)-C_{Y 4}\left(\delta_{R U D T}+\delta_{R U D B}\right)\right] \\
& Z_{a}=-Q_{\infty}\left[C_{Z 1} \cos (\alpha / 2) \sin (2 \alpha)+C_{Z 2} \sin (2 \alpha)+C_{Z 3} \sin \alpha \sin (|\alpha|)+C_{Z 4}\left(\delta_{E L V L}+\delta_{E L V R}\right)\right] \\
& L_{a}=Q_{\infty}\left[C_{L 1}\left(\delta_{E L V L}-\delta_{E L V R}+\delta_{R U D T}-\delta_{R U D B}\right)+C_{L 2} \sin \beta \sin (|\beta|)\right] \\
& M_{a}=-Q_{\infty}\left[C_{M 1} \cos (\alpha / 2) \sin (2 \alpha)+C_{M 2} \sin (2 \alpha)+C_{M 3} \sin \alpha \sin (|\alpha|)+C_{M 4}\left(\delta_{E L V L}+\delta_{E L V R}\right)\right] \\
& N_{a}=Q_{\infty}\left[C_{N 1} \cos (\beta / 2) \sin (2 \beta)+C_{N 2} \sin (2 \beta)+C_{N 3} \sin \beta \sin (|\beta|)-C_{N 4}\left(\delta_{R U D T}+\delta_{R U D B}\right)\right]
\end{aligned}
$$

where $Q_{\infty}=0.5 \rho V^{2}, \alpha$ and $\beta$ are angle of attack and sideslip angle respectively, $\delta_{\mathrm{ELVL}}$ and $\delta_{\mathrm{ELVR}}$ are elevator deflection angles, $\delta_{\text {RUDT }}$ and $\delta_{\text {RUDB }}$ are rudder angles, $C_{* *}$ are coefficients of aerodynamic forces determined by the profile parameters shown in Figure 1.

Due to the large volume/weight ratio of the airship, added inertia forces should be considered in modeling as a result of that an accelerating or decelerating airship must move (or deflect) some volume of surrounding air as it moves through it [7]. 


$$
\boldsymbol{F}_{a d d}=-\rho \nabla\left[\begin{array}{l}
k_{1} \dot{u}-k_{2} v r+k_{2} w q \\
k_{2} \dot{v}+k_{1} u r-k_{2} w p \\
k_{2} \dot{w}-k_{1} u q+k_{2} v p
\end{array}\right], \quad \boldsymbol{M}_{a d d}=-\rho \nabla\left[\begin{array}{c}
0 \\
k_{3} \dot{q}-k_{3} p r \\
k_{3} \dot{r}+k_{3} p q
\end{array}\right]
$$

where $k_{1}, k_{2}$ and $k_{3}$ are ellipsoid inertia factors, $[u, v, w]^{\mathrm{T}}$ and $[p, q, r]^{\mathrm{T}}$ are linear and angular velocities in $O x y z, \nabla$ is the volume of airship.

Generally speaking, thrust provided to airship is from propellers. There are many ways to the layout of the propellers, in this paper the propellers are installed on left and right sides of gondola with vector regulation [8]. Suppose the value of thrust is $T$ and the inclination angle is $\mu$, then the thrust and the torques from thrust are as follows.

$$
\boldsymbol{F}_{T}=\left[\begin{array}{c}
2 T \cos \mu \\
0 \\
2 T \sin \mu
\end{array}\right], \quad \boldsymbol{M}_{T}=\left[\begin{array}{c}
-T y_{p} \sin \mu \\
T z_{p} \cos \mu-T x_{p} \sin \mu \\
+T y_{p} \cos \mu
\end{array}\right]
$$

where $\left[x_{p}, y_{p}, z_{p}\right]^{\mathrm{T}}$ is the position of propeller as shown in Figure 1.

Dynamic equations are established through the geometrical relations between the motion parameters [8]. The equations of motion are used to describe the change of position and attitude of airship. And the development of the equations of motion follows standard aircraft practice, the equations of attitude motion are as follows.

$$
\left[\begin{array}{c}
\dot{\phi} \\
\dot{\theta} \\
\dot{\psi}
\end{array}\right]=\left[\begin{array}{ccc}
1 & \tan \theta \sin \phi & \tan \theta \cos \phi \\
0 & \cos \phi & -\sin \phi \\
0 & \sin \phi / \cos \theta & \cos \phi / \cos \theta
\end{array}\right]\left[\begin{array}{l}
p \\
q \\
r
\end{array}\right]
$$

where $[\theta, \Psi, \Phi]^{\mathrm{T}}$ is the attitude angle between $O x_{g} y_{g} z_{g}$ to $O x y z$. Suppose the altitude of airship $h=z_{g}$, the equations of position motion are as follows.

$$
\left[\begin{array}{c}
\dot{x}_{g} \\
\dot{y}_{g} \\
\dot{h}
\end{array}\right]=\left[\begin{array}{ccc}
\cos \theta \cos \psi & \sin \theta \cos \psi \sin \phi-\sin \psi \cos \phi & \sin \theta \cos \psi \cos \phi+\sin \psi \sin \phi \\
\cos \theta \sin \psi & \sin \theta \sin \psi \sin \phi+\cos \psi \cos \phi & \sin \theta \sin \psi \cos \phi-\cos \psi \sin \phi \\
\sin \theta & -\cos \theta \sin \phi & -\cos \theta \cos \phi
\end{array}\right]\left[\begin{array}{c}
u \\
v \\
w
\end{array}\right]
$$

Suppose $[X, Y, Z]^{\mathrm{T}}$ and $[L, M, N]^{\mathrm{T}}$ are the total force and moment applied on the airship, the dynamic equations are as follows.

$$
\left[\begin{array}{c}
I_{x} \dot{p}+\left(I_{z}-I_{y}\right) q r-I_{x z}(\dot{r}+p q)-m z_{c}(\dot{v}+u r-w p) \\
I_{y} \dot{q}+\left(I_{x}-I_{z}\right) p r+I_{x z}\left(p^{2}-r^{2}\right)+m z_{c}(\dot{u}+w q-v r)-m x_{c}(\dot{w}+v p-u q) \\
I_{z} \dot{r}+\left(I_{y}-I_{x}\right) p q+I_{x z}(q r-\dot{p})+m x_{c}(\dot{v}+u r-w p) \\
m(\dot{u}-v r+w q)-m x_{c}\left(q^{2}+r^{2}\right)+m z_{c}(\dot{q}+p r) \\
m(\dot{v}+u r-w p)+m x_{c}(\dot{r}+p q)+m z_{c}(q r-\dot{p}) \\
m(\dot{w}+v p-u q)+m x_{c}(r p-\dot{q})-m z_{c}\left(q^{2}+p^{2}\right)
\end{array}\right]=\left[\begin{array}{c}
L \\
M \\
N \\
X \\
Y \\
Z
\end{array}\right]
$$

The equations of motion, (11) and (12), may be assembled together to describe the fully coupled dynamic behavior of the airship. This model would be most appropriate for computer simulation but is not very amenable to mathematical analysis, which is more readily accomplished with a linearized small perturbation model.

\section{Dynamic Stability Analysis}

The state equation, usually in concise form, is readily solved to obtain the response transfer functions [9]. Because the solution involves algebraic manipulation of matrices, it is necessary to first obtain the Laplace transform of the state equation thus, assuming zero initial conditions [10].

$$
s \boldsymbol{x}(s)=\boldsymbol{A x}(s)+\boldsymbol{B u}(s)
$$


It is reasonable to assume zero initial conditions because the motion of interest is defined by small perturbations about a steady datum trim state. The solution of the state equation follows readily,

$$
\boldsymbol{x}(s)=(s \boldsymbol{I}-\boldsymbol{A})^{-1} \boldsymbol{B} \boldsymbol{u}(s)=\boldsymbol{G}(s) \boldsymbol{u}(s)
$$

where $\boldsymbol{I}$ is a unit matrix and $\boldsymbol{G}(s)$ is the transfer function matrix, and is typically of the form [11]

$$
\boldsymbol{G}(s)=\frac{\boldsymbol{N}(s)}{\Delta(s)}
$$

where, $\Delta(s)=\operatorname{det}(s \boldsymbol{I}-\boldsymbol{A})$ is the characteristic polynomial and common denominator of the transfer functions [12]. Also $\Delta(s)=0$ defines the characteristic equation whose zeros, or, equivalently, eigenvalues of $\boldsymbol{A}$, provide a complete description of stability.

Suppose a neutrally buoyant nonrigid airship of mass $86800 \mathrm{~kg}$ flying at a speed of $20 \mathrm{~m} / \mathrm{s}$ at altitude of $22 \mathrm{~km}$ [9]. The approximate stability modes compare with the exact solution as shown in Table 1.

Table.1. Longitudinal stability modes

\begin{tabular}{ccccc}
\hline & \multicolumn{4}{c}{ Neutral Buoyancy $-86800 \mathrm{~kg}$ Mass $-20 \mathrm{~m} / \mathrm{s}-$ altitude $22 \mathrm{~km}$} \\
\hline Longitudinal & \multicolumn{2}{c}{ Exact Solution } & \multicolumn{2}{c}{ Approximate Solution } \\
\cline { 2 - 5 } Stability Mode & Roots & Characteristic & Roots & Characteristic \\
\hline Surge & $(s+0.0226)$ & Stable & $(s+0.0234)$ & Stable \\
Heave & $(s+0.8634)$ & Stable & $(s+0.8625)$ & Stable \\
Pendulum & $\left(s^{2}+0.157 s+0.148\right)$ & Stable & $\left(s^{2}+0.157 s+0.148\right)$ & Stable \\
\hline
\end{tabular}

The approximate stability modes compare with the exact solution as shown in Table 2.

Table.2. Lateral stability modes

\begin{tabular}{ccccc}
\hline \multicolumn{5}{c}{ Neutral Buoyancy $-86800 \mathrm{~kg}$ Mass $-20 \mathrm{~m} / \mathrm{s}-$ altitude $22 \mathrm{~km}$} \\
\cline { 2 - 5 } Lateral & \multicolumn{2}{c}{ Exact Solution } & \multicolumn{2}{c}{ Approximate Solution } \\
\cline { 2 - 5 } Stability Mode & Roots & Characteristic & Roots & Characteristic \\
\hline Yaw subsidence & $(s+0.893)$ & Stable & $(s+0.904)$ & Stable \\
Sideslip & $(s+0.128)$ & Stable & $(s+0.1252)$ & Stable \\
subsidence & $\left(s^{2}+0.293 s+0.543\right)$ & Stable & $\left(s^{2}+0.285 s+0.551\right)$ & Stable \\
Roll oscillation & $\left(s^{2}\right)$ &
\end{tabular}

Clearly, for both longitudinal and lateral dynamics, the approximate and exact stability modes compare very well. However, the approximate model is not particularly useful for producing a numerical solution, although this may be helpful for confirming the output of a computer program.

\section{Control and Response of Airship}

To illustrate the control and response characteristics of the airship, a simulation is performed. The baseline airship design parameters are presented in Table 3. The geometric dimensions and aerodynamic properties are designed to be similar with those illustrated by Lee and Bang [13].At the pressure altitude where the internal helium bags expand to the largest size, suppose that the buoyancy equals to the gravity and environment is steady, and helium leak is ignored.

Table.3. Baseline airship design parameters

\begin{tabular}{ll}
\hline Design parameter & \multicolumn{1}{c}{ Value } \\
\hline Maximum airspeed & $30 \mathrm{~m} / \mathrm{s}$ \\
Pressure altitude & $22 \mathrm{~km}$ \\
Length & $1200 \mathrm{~m}$ \\
Envelop volume & $265,746 \mathrm{~m}^{3}$ \\
Hull area & $25,564 \mathrm{~m}^{2}$ \\
Weight of envelope & $6,385 \mathrm{~kg}$ \\
Maximum take-off weight & $323,986 \mathrm{~kg}$ \\
\hline
\end{tabular}

The longitudinal response to elevator is shown in Figure 2, for which the input command is a 0.2 rad $\left(11.5^{\circ}\right)$ elevator step such as to cause a nose-down response. In aerodynamic terms the input is relatively large, as a deflection angle in the range 0.26 to 0.35 rad would cause a flap-type control 
surface to stall, with the consequent loss of effectiveness. The magnitudes of the response variables are very small and the time taken for the transient to settle is in the order of one minute. This clearly demonstrates a relatively low longitudinal control power and a rather sluggish response characteristic.
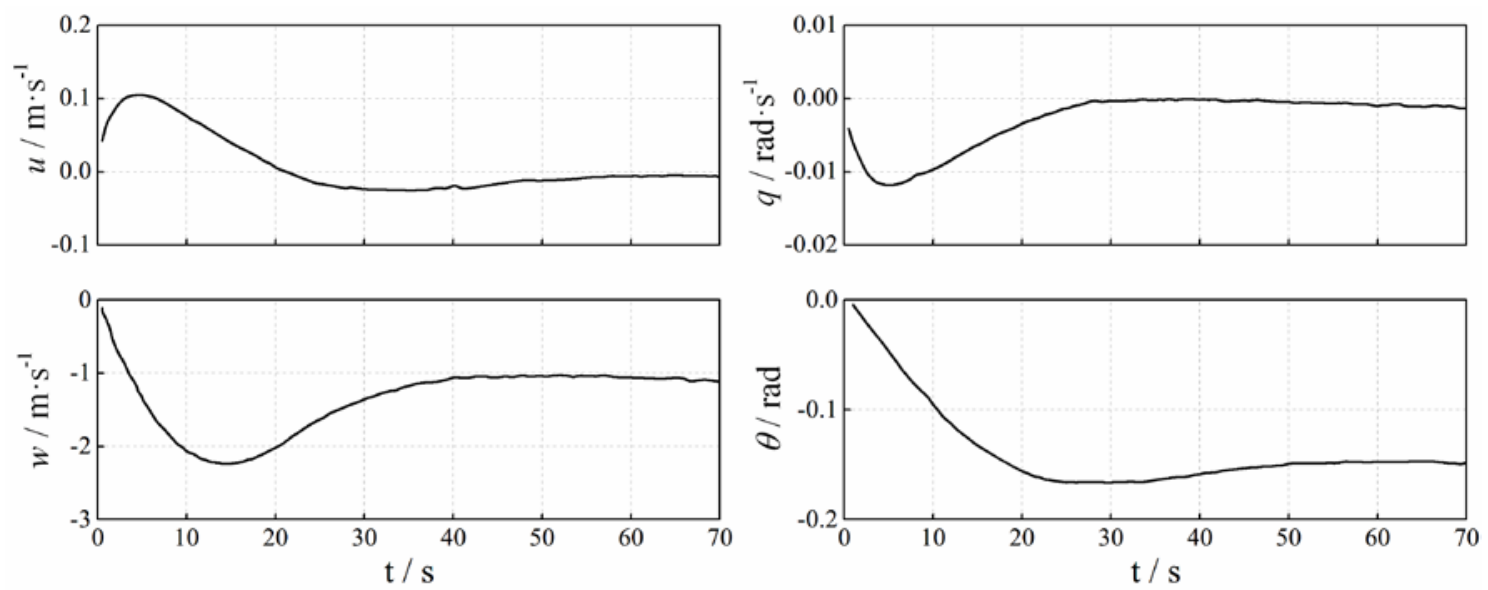

Fig.2. Longitudinal response to a 0.2-rad elevator step input

The longitudinal response to a 5-kN step increase in thrust is shown in Figure 3. It is clear that, although the engines are mounted well below the center of gravity, the pitch response to a thrust change is very small. The only signification response is in velocity $\mathrm{u}$, as might be expected. Again, the general magnitude and time scale of response confirms that longitudinal control power is low and response is sluggish.
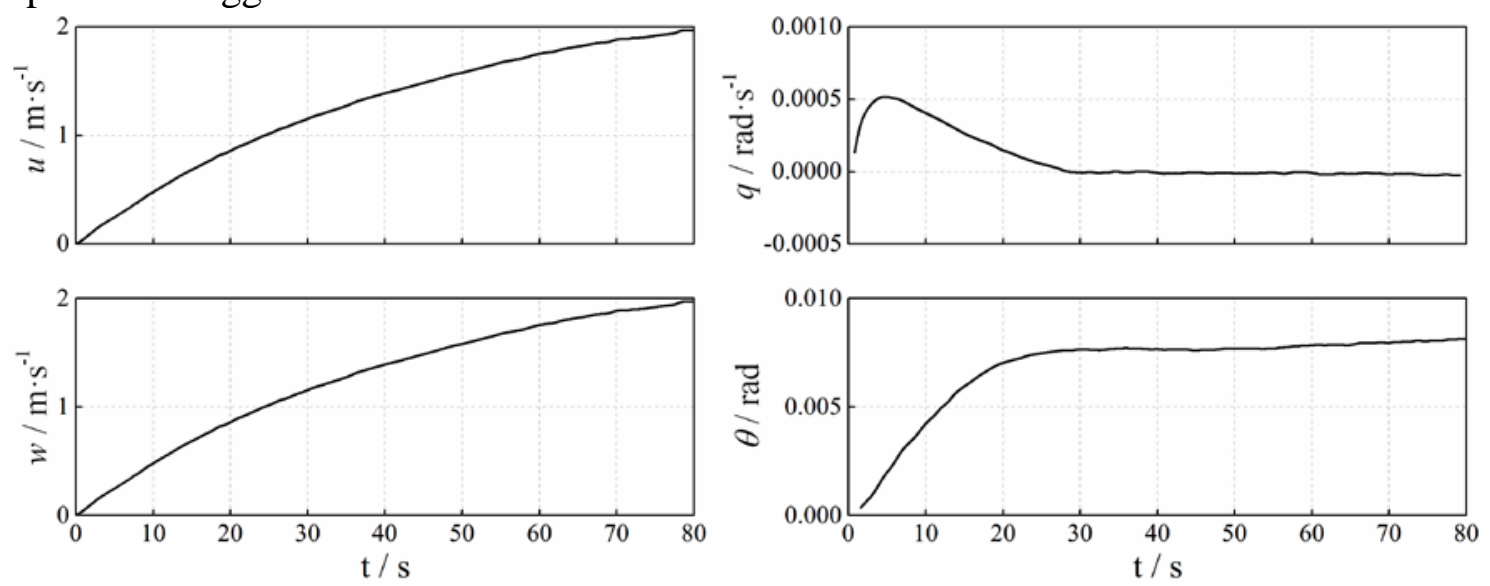

Fig.3. Longitudinal response to a 5-kN step increase in thrust

The lateral response to a 0.2-rad step command input to rudder is shown in Figure 4. In aerodynamic term, this is a relatively large input and as the response magnitudes are very small, it is clear that rudder control power is low. The transients, however, settle in approximately 30 seconds, indicating that lateral response is a little less sluggish than longitudinal response.
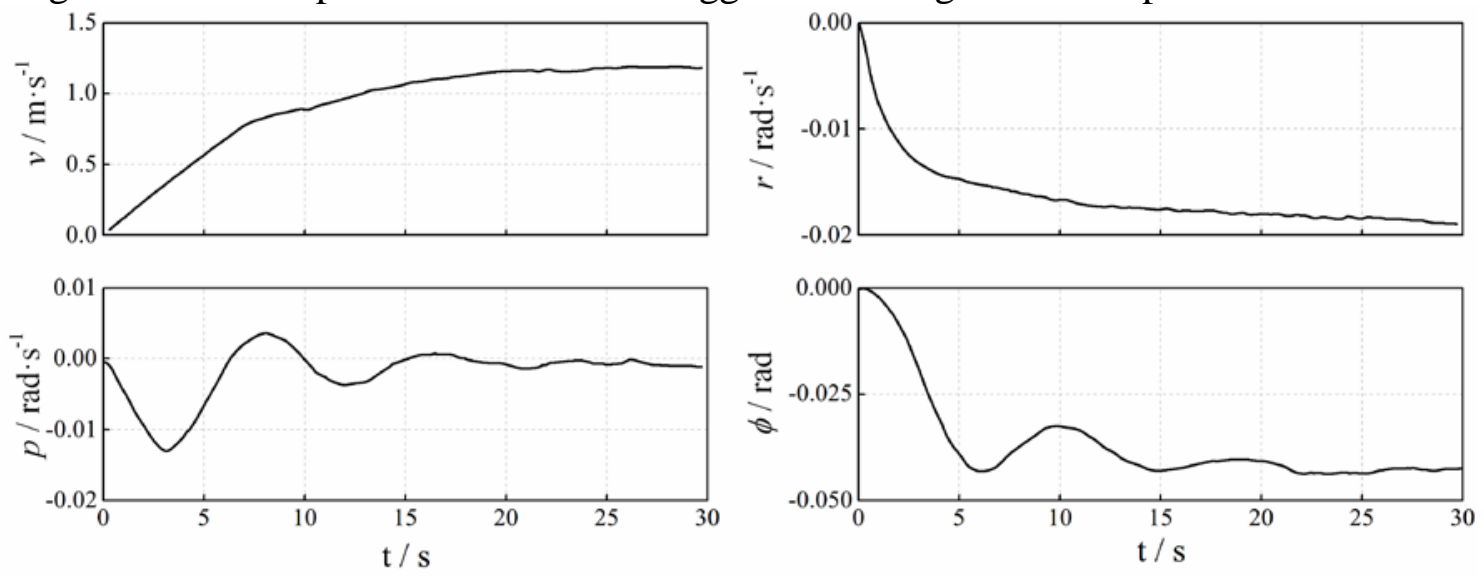

Fig.4. Lateral response to a 0.2-rad rudder step input 


\section{Conclusion}

Based on the particular properties of stratospheric airship, dynamic models and kinematic equations are addressed. Dynamic stability analysis results show that the airship is stable in specific conditions. The surge, heave and pendulum mode are main modes in longitudinal direction. And yaw subsidence, sideslip subsidence and oscillatory roll mode are main modes in lateral direction. For all practical purposes, the airship appears to the pilot to be neutrally stable at hover, with the only significantly visible response dynamics being the undamped oscillatory.

\section{References}

[1] Xiao Guo, Ming Zhu. Ascent trajectory optimization for stratospheric airship with thermal effects [J]. Advances in Space Research, 2013, 52 (6): 1097-1110.

[2] Paiva Ely Carneiro de, Bueno Samuel Siqueira, Bergerman Marcel. A robust Pitch Attitude Controller for Aurora’s Semi-Autonomous Robotic Airship [C]. AIAA Lighter-Than-Air Systems Technology Conference. Reston: AIAA, 1999: 1-8.

[3] Acosta D M, Joshi S S. Adaptive Nonlinear Dynamic Inversion Control of an Autonomous Airship for the Exploration of Titan [C]. AIAA Guidance, Navigation and Control Conference and Exhibit. Reston: AIAA, 2007: 1-13.

[4] Hygounenc E, Soueres P. Automatic Airship Control Involving Backstepping Techniques [C]. IEEE International Conference on Systems, Man and Cybemetics. Reston: IEEE, 2002: 1-6.

[5] Trevino R, Frye M, Franz J A. Robust Receding Horizon Control of A Tri-Turbofan Airship [C]. 2007 IEEE International Conference on Control and Automation. Piscataway: IEEE, 2007: 671-676.

[6] LI Z B, WU L, ZHANG J R, etal. Review of dynamics and control of stratospheric airships [J]. Advances in Mechanics, 2012, 2012(4): 482-493.

[7] Liu D, Wang X L, Shan X X. Added Mass to Stratospheric Airship and Its Effect on Motion [J]. Computer Simulation, 2006, 23(6): 52-56.

[8] Sun D Y, Yi W J. Design of Decoupling Control System for Glide Extended Range Guided Munition Based on PID Neural Network [J]. Computing Technology and Automation, 2011, 30(2): 21-25.

[9] G Khoury. Airship Technology [M]. London: Cambridge University Press, 2004.

[10] Xiao J, Zhang W W, Guo X P, etal. Design of pressure control system for airship based on fuzzy PID [J]. Journal of Liaoning Technical University (Natural Science), 2010, 29(5): 807-809.

[11] Mueller J B, Zhao Y J, Garrard W L. Optimal ascent trajectories for stratospheric airships using wind energy [J]. Journal of Guidance, Control, and Dynamics. 2009a, 32 (4): 1232-1245.

[12] Colozza A, Dolce J L. High-altitude, long-endurance airships for coastal surveillance [R]. NASA Technical Report, NASA/TM-2005-213427, 2005.

[13] Lee S, Bang H. Three-dimensional ascent trajectory optimization for stratospheric airship platforms in the jet stream. Journal of Guidance, Control and Dynamics, 2007, 30(5): 1341-1352. 